\title{
Effects of Solar Particle Event-Like Proton Radiation and/or Simulated Microgravity on Circulating Mouse Blood Cells
}

\author{
Ana L. Romero-Weaver ${ }^{1}$, Liyong Lin ${ }^{1}$, Alejandro Carabe-Fernandez ${ }^{1}$, and Ann R. Kennedy ${ }^{1}$ \\ ${ }^{1}$ Department of Radiation Oncology, University of Pennsylvania School of Medicine, Philadelphia, \\ Pennsylvania
}

\begin{abstract}
Astronauts traveling in space missions outside of low Earth orbit will be exposed for longer times to a microgravity environment. In addition, the increased travel time involved in exploration class missions will result in an increased risk of exposure to significant doses of solar particle event (SPE) radiation. Both conditions could significantly affect the number of circulating blood cells. Therefore, it is critical to determine the combined effects of exposure to both microgravity and SPE radiation. The purpose of the present study was to assess these risks by evaluating the effects of SPE-like proton radiation and/or microgravity, as simulated with the hindlimb unloading (HU) system, on circulating blood cells using mouse as a model system. The results indicate that exposure to $\mathrm{HU}$ alone caused
\end{abstract}

Key words: Proton Radiation; Simulated Microgravity; Hematopoietic Cells

Correspondence to: Ann R. Kennedy University of Pennsylvania Perelman Center for Advanced Medicine Department of Radiation Oncology

195 John Morgan Building

3620 Hamilton Walk

Philadelphia, PA 19104

Telephone: 215-898-0079

E-mail: akennedy@mail.med.upenn.edu minimal or no significant changes in mouse circulating blood cell numbers. The exposure of mice to SPE-like proton radiation with or without HU treatment caused a significant decrease in the number of circulating lymphocytes, granulocytes and platelets. The reduced numbers of circulating lymphocytes, granulocytes, and platelets, resulting from the SPE-like proton radiation exposure, with or without HU treatment, in mice suggest that astronauts participating in exploration class missions may be at greater risk of developing infections and thrombotic diseases; thus, countermeasures may be necessary for these biological endpoints.

\section{INTRODUCTION}

Manned spaceflight missions beyond low Earth orbit are planned for the near future. During such missions, astronauts will be exposed to several stressors, including reduced gravity environments that also involve exposure to space radiations. Of particular concern is potential astronaut exposure to significant doses of radiation associated with solar particle events (SPEs), which are sporadic bursts of energetic charged particles, consisting mostly of low energy protons emitted from magnetically disturbed regions of the Sun (Hellweg and BaumstarkKhan, 2007). It has been calculated that astronauts exposed to SPE radiation during extravehicular activities can receive a deep tissue dose of up to 2 Gy (Kim et al., 2009; Townsend, 2002). Exposure 
to these space stressors may affect several organs. Cells of the innate immune system are especially sensitive to both, reduced gravity and radiation exposure (Dainiak, 2002; Plett et al., 2004).

Microgravity exposure of human and/or animals during spaceflight missions, or in Earth based simulated microgravity experiments, have shown both, increases and decreases in the numbers of several types of immune cells in the circulation (Allebban et al., 1994; Ichiki et al., 1996; Borchers et al., 2002; Kaur et al., 2005). The majority of these reports show either a decrease or no change in the number of circulating lymphocytes, and an increase in the number of circulating neutrophils and monocytes. Exposure to microgravity or simulated microgravity also causes alterations in the function of some blood cells, such as lymphocytes (Sanzari et al., 2011; Wei et al., 2003), platelets (Dai et al., 2009), and neutrophils (Murdaca et al., 2003; Kaur et al., 2004). Decreased numbers of all blood cell counts have been observed in mice exposed to SPE-like proton radiation simulated with low energy protons (Gridley et al., 2008; Maks et al., 2011; Romero-Weaver et al., 2013a).

The purpose of this study was to evaluate the combined effects of reduced gravity and SPE radiation on blood cell counts using the mouse model. SPE radiation was simulated with low energy protons (SPE-like proton radiation) and microgravity was simulated with the HU system (Chapes et al., 1993; Morey-Holton and Globus, 2002). Their individual and combined effects were determined at different proton doses in blood cells of mice at nadir times that have been previously determined after exposing mice to SPE-like proton radiation (Romero-Weaver et al., 2013a). The effective dose (ED) of radiation needed to decrease cell counts by $10 \%, 50 \%$, and $90 \%$ ( $\mathrm{ED}_{10}, \mathrm{ED}{ }_{50}$, and $\mathrm{ED}_{90}$, respectively) was also determined. Finally, the kinetics of the combined effects of HU and 2 Gy SPE-like proton radiation were obtained for lymphocytes, granulocytes, and platelets.

There is little information in the literature about the combined effects of reduced gravity and SPE radiation on blood cell counts (Sanzari et al., 2013). In our previous report on this topic (Sanzari et al., 2013), the studies were focused primarily on the effects of a single dose level (2 Gy) of SPE-like proton radiation, with or without
HU, on lymphocyte subtype populations and activity, but they did provide some information about total white blood cell counts on days four and 21 post-irradiation in mice exposed to SPElike proton radiation with or without HU. The present study utilized similar exposure conditions for the mice, but provides new data on the effects of three different doses of SPE-like proton radiation $(0.5,1.0$, and 2.0 Gy) at more time points than those evaluated previously. In addition, these studies were performed to determine whether there was evidence of interaction between the two different stressors (proton radiation and $\mathrm{HU}$ ) on the responses of the blood cells at various time points.

\section{MATERIAL AND METHODS}

\section{Mice}

Female imprinting control region (ICR) mice at six weeks of age were purchased from Taconic Farms Inc. (Germantown, NY). Animals were acclimated for seven days in the University of Pennsylvania animal facility. Five animals were housed per cage with ad libitum access to water and food pellets during the acclimation period. Animals were then caged individually, as described below. The animal care and treatment procedures were approved by the Institutional Animal Care and Use Committee of the University of Pennsylvania.

\section{Experimental Groups}

Mice were divided into the following groups: 1) Sham-controls (sham-HU and shamirradiation); 2) 0.5 Gy proton radiation with sham$\mathrm{HU}$; 3) 1.0 Gy proton radiation with sham-HU; 4) 2.0 Gy proton radiation with sham-HU; 5) $\mathrm{HU}$ alone; 6) $\mathrm{HU}+0.5$ Gy proton radiation; 7) $\mathrm{HU}+$ 1.0 Gy proton radiation; and 8) $\mathrm{HU}+2.0 \mathrm{~Gy}$ proton radiation.

\section{Simulated Microgravity by Hindlimb- Unloading (HU)}

After a week of acclimation and two days before irradiation, mice were exposed to $\mathrm{HU}$ with some variations of the method reported by MoreyHolton and Globus (Morey-Holton and Globus, 2002). Mice were individually housed in custom made plexiglass cages. The top and bottom of each cage contains holes for aeration and for 
elimination of droppings and urine. Before suspension, mice were weighed. For suspension, the base of the mouse tail was cleaned with isopropyl alcohol and then Benzoin tincture (Ruger Chemical Co., Inc., Linden, NJ) was applied. After it dried, a piece of Steri-Strip ${ }^{\mathrm{TM}}$ tape (3M, St. Paul, MN) was wrapped around the tail and the tail was attached to a metal chain using athletic tape. The mouse was suspended at a $30^{\circ}$ head-down tilt by adjusting the chain length using a hook attached to a metal rod placed at the top of the cage. At this angle, the mouse had no load bearing on the hindlimbs; thus, the body weight was supported primarily by the front limbs. The hook can move along the rod and rotate 360 degrees with minimal friction, which allows free movement of the mouse to all areas of the cage; this arrangement allows the mouse to have free access to water and food.

Food was randomly placed on the cage floor and a water bottle was attached outside of the cage with the metal tube that provides the water inserted into the cage. A piece of Nestlets ${ }^{\mathrm{TM}}$ (Ancare, Bellmore, NY) was placed in each cage for environmental enrichment. An absorbent pad was placed under the cage to collect droppings and urine. Food, water, and absorbent pads were changed daily, the average food consumption was determined, and the animals were observed twice a day to ensure that they were properly fed and hydrated, that they remain suspended at the right angle, and that their tails were not damaged. Overall, all mice were maintained at the right angles and none of them lost more than $10 \%$ of their body weight compared with their weight before suspension. Control mice without exposure to HU (sham-HU) were individually housed in similar conditions except that they were not suspended. Sham-HU mice were pair-fed according to the average consumption of the suspended mice.

\section{Radiation}

Two days after exposure to $\mathrm{HU}$, mice were restrained in custom designed Plexiglass chambers and exposed to total body proton radiation at doses of $0.5,1$, or 2 Gy administered at a dose rate of $0.5 \mathrm{~Gy} /$ minute. Proton irradiation was carried out as previously described (RomeroWeaver et al., 2013b). Sham-irradiated control mice (corresponding to the sham-HU control mice) were restrained in Plexiglas chambers, but the mice were not irradiated. All mice were maintained in the chambers for the same amount of time (approximately 20 minutes). After irradiation, mice were returned to their corresponding HU cages and the groups exposed to HU treatment were re-suspended.

\section{Blood Sample Collection and Processing}

On days 2, 4, 10, and 16 post-irradiation, which corresponds to days $4,6,12$, and 16 days, respectively, after the initiation of HU treatment, five mice from each group were euthanized by $\mathrm{CO}_{2}$ inhalation. Blood samples were collected by cardiac puncture, placed into lavender top blood BD microtainer collection tubes containing EDTA (BD, Franklin Lakes, NJ) and sent to an Antech Diagnostics facility (Lake Success, NY) for complete automated blood cell count analyses using a Cell-Dyn 3700 multiparameter automated hematology analyzer. Each result was confirmed by a technologist who performed manual counts of blood smears.

\section{Data and Statistical Analyses}

The mean counts of lymphocytes, granulocytes (neutrophils + eosinophils + basophils), and platelets of the no treatment control group were calculated and used as the baseline control values for the respective blood cell types. For each animal at each time point after irradiation, the count of each blood cell type was divided by the respective baseline control value and the result was expressed as a fraction of the control value for further analyses. The relationship between the radiation dose and results of each blood cell type were determined at each nadir time after irradiation by fitting the data to a natural $\log$ linear model $\left(y=e^{-a D}\right)$, via non-linear regression analysis using the radiation dose (D) as the independent variable, and cell count expressed as the fraction of control (y) as the dependent variable.

The ED required to reduce the cell count by $10 \% \quad\left(\mathrm{ED}_{10}\right), \quad 50 \% \quad\left(\mathrm{ED}_{50}\right), \quad 90 \% \quad\left(\mathrm{ED}_{90}\right)$, and associated $95 \%$ confidence intervals $(95 \% \mathrm{CI})$ were calculated for the SPE-like proton irradiated groups, with or without HU. The non-linear regression analysis and $\mathrm{ED}_{10}, \mathrm{ED}_{50}$, and $\mathrm{ED}_{90}$ calculation were performed using STATA statistical software (Stara/IC 12.1). The fitted 
$\mathrm{ED}_{10}, \mathrm{ED}_{50}$, and $\mathrm{ED}_{90}$ values for the $\mathrm{HU}$ treatment groups were compared with the $95 \%$ confidence interval for $\mathrm{ED}_{10}, \mathrm{ED}_{50}$, and $\mathrm{ED}_{90}$ values of the groups without $\mathrm{HU}$ treatment to determine whether $\mathrm{ED}_{10}, \mathrm{ED}_{50}$, and $\mathrm{ED}_{90}$ values were significantly different between the groups with and without HU treatment. If a fitted ED value for the HU groups is above (or below) the upper (or lower) limit of the $95 \%$ confidence interval for the groups without $\mathrm{HU}$, then the ED value for the HU group is significantly higher (or lower) than the corresponding ED value for the group without $\mathrm{HU}$ at $\mathrm{p}<0.05$ (i.e., $1-0.95=0.05$ ).

The main effects of Dose, HU, and their interactions were determined by a design of experiments (DOE) approach using a general linear model $[\mathrm{y}=\mathrm{f}($ Dose, $\mathrm{HU}$, Dose·HU)] with Dose and HU (yes or no) as independent variables. DOE analyses were performed using Minitab statistical software, release 15 (Minitab, Inc., State College, PA). For the time course comparison, group means at different time points were analyzed by one-way analysis of variance (ANOVA), followed by Tukey's test to compare the results for each group at each time point with the corresponding sham irradiated control values. In addition, a $t$-test was utilized to compare the results of matching groups with or without HU treatment at each time point. Both, one-way ANOVA and the $t$-test were done using SigmaPlot 12.

\section{RESULTS}

The main effects and interactions of SPE-like proton radiation dose and $\mathrm{HU}$ treatment were determined for lymphocytes, granulocytes, and platelets by DOE analyses at previously reported nadir times (Romero-Weaver et al., 2013a); these nadir times are as follows: two days postirradiation for lymphocytes, four and 16 days post-irradiation for granulocytes, and 10 days post-irradiation for platelets. The results indicated that at the lymphocyte nadir time, the cell counts decreased significantly in a dose dependent manner (Figure 1A left panel, $\mathrm{F}=26.47, \mathrm{p}<$ $0.001)$ and were significantly higher in mice with $\mathrm{HU}$ treatment than without HU treatment (Figure $1 \mathrm{~A}$ right panel, $\mathrm{F}=12.02, \mathrm{p}=0.002$ ). There was no significant interaction between the radiation dose and HU treatment (Figure 1B, F = 2.22, p = 0.108).
The DOE analysis also demonstrated that the granulocyte counts decreased significantly in a dose dependent manner at both the first (Figure 2A left panel, $\mathrm{F}=7.04, \mathrm{p}<0.001)$ and the second (Figure 3A left panel, $\mathrm{F}=6.80, \mathrm{p}=0.001$ ) nadir times, respectively. The granulocyte counts were not significantly affected by HU treatment at the first nadir time (Figure 2A right panel, $\mathrm{F}=0.39, \mathrm{p}$ $=0.539$ ) but were significantly higher in animals with HU treatment at the second nadir time (Figure $3 \mathrm{~A}$ right panel, $\mathrm{F}=11.62, \mathrm{p}=0.002$ ). No significant interaction was found between the radiation dose and $\mathrm{HU}$ treatment for granulocyte counts at either the first (Figure $2 \mathrm{~B}, \mathrm{~F}=0.17, \mathrm{p}=$ 0.915 ), or the second (Figure $3 \mathrm{~B}, \mathrm{~F}=2.26, \mathrm{p}=$ 0.103 ) nadir times.

The DOE analysis for platelets indicated the cell counts decreased significantly in a dose dependent manner (Figure 4A left panel, $\mathrm{F}=4.98$, $p=0.007$ ), but were not significantly affected by HU treatment (Figure 4A right panel, $\mathrm{F}=2.16, \mathrm{p}$ $=0.153)$. There was no significant interaction between the radiation dose and HU treatment on the platelet counts (Figure $4 \mathrm{~B}, \mathrm{~F}=1.38, \mathrm{p}=$ 0.268).

$\mathrm{ED}_{10}, \mathrm{ED}_{50}$, and $\mathrm{ED}_{90}$ values were calculated for each blood cell type at each nadir, and the results are shown in Table 1. In the case of lymphocytes, the fitted values of $\mathrm{ED}_{10}, \mathrm{D}_{50}$, and $\mathrm{ED}_{90}$ for the irradiated mice with $\mathrm{HU}$ treatment was $0.23,1.49$, and 4.93 Gy, respectively, which were above the upper limit of the $95 \%$ confidence intervals of the $\mathrm{ED}_{10}, \mathrm{ED}_{50}$, and $\mathrm{ED}_{90}$ value for the irradiated mice without HU treatment. Collectively, these results suggest that the combined treatment with radiation and HU is less effective in reducing lymphocyte counts than radiation without HU. In the case of granulocytes on day four post-irradiation, the fitted values of ED were not significantly different between irradiated groups with and without HU treatment $(p>0.05)$, since the ED values for the irradiated groups with HU falls within the $95 \%$ confidence intervals for the corresponding ED values for the irradiated group without HU.

The dose response curve slope for granulocytes on day 16 post-irradiation, with the combined treatment of radiation and $\mathrm{HU}$, was not significantly different from zero; therefore, meaningful $\mathrm{ED}_{10}, \mathrm{ED}_{50}$, and $\mathrm{ED}_{90}$ values could not be calculated, which indicate that radiation, in 
combination with HU had less of a reducing effect on granulocyte counts than did radiation alone. It also indicated that radiation in combination with HU had less of a reducing effect on granulocyte cell counts on day 16, than on day 4 postirradiation. For platelet counts on day 10 after irradiation, the $\mathrm{ED}_{10}, \mathrm{ED}_{50}$, and $\mathrm{ED}_{90}$ values were comparable between the irradiated groups with or without $\mathrm{HU}(\mathrm{p}>0.05)$. Since fitted $\mathrm{ED}_{50}$ values were below 2 Gy for all blood cell types analyzed, except for granulocytes at the second nadir time, these results indicate that exposure to a dose of 2 Gy of protons alone, or in combination with HU, decreased the number of lymphocytes, granulocytes, and platelets by more than $50 \%$ at the specified nadir times.

Table 1. Fitted value (FV) of the effective dose of radiation (ED) to decrease the cell count by $10 \%\left(E_{10}\right)$, $50 \%\left(E D_{50}\right)$ and $90 \%\left(E D_{90}\right)$ in mice exposed to SPE-like proton and/or HU.

\begin{tabular}{|c|c|c|c|c|c|c|}
\hline & \multicolumn{2}{|c|}{ ED10 } & \multicolumn{2}{|c|}{ ED50 } & \multicolumn{2}{|c|}{ ED90 } \\
\hline & FV (Gy) & 95\% CI* & FV (Gy) & $95 \%$ CI & FV (Gy) & $95 \%$ CI \\
\hline \multicolumn{7}{|c|}{ Lymphocytes } \\
\hline \multicolumn{7}{|c|}{ day-2 post-irradiation } \\
\hline No HU & 0.13 & $0.09-0.16$ & 0.83 & $0.58-1.08$ & 2.76 & $1.92-3.60$ \\
\hline HU & 0.23 & $0.09-0.37$ & 1.49 & $0.56-2.41$ & 4.93 & $1.87-8.00$ \\
\hline \multirow{2}{*}{\multicolumn{7}{|c|}{$\begin{array}{c}\text { Granulocytes } \\
\text { day-4 post-irradiation }\end{array}$}} \\
\hline & & & & & & \\
\hline No HU & 0.24 & $0.07-0.40$ & 1.56 & $0.48-2.64$ & 5.19 & $1.59-8.79$ \\
\hline $\mathrm{HU}$ & 0.29 & $0.16-0.41$ & 1.88 & $1.06-2.71$ & 6.25 & $3.52-8.99$ \\
\hline \multirow{2}{*}{\multicolumn{7}{|c|}{$\begin{array}{c}\text { Granulocytes } \\
\text { day-16 post-irradiation }\end{array}$}} \\
\hline & & & & & & \\
\hline No HU & 0.17 & $0.10-0.24$ & 1.14 & $0.68-1.60$ & 3.78 & $2.25-5.32$ \\
\hline $\mathrm{HU}$ & $\mathrm{NM}^{* *}$ & $\mathrm{NM}$ & NM & $\mathrm{NM}$ & NM & NM \\
\hline \multicolumn{7}{|c|}{$\begin{array}{c}\text { Platelets } \\
\text { day-10 post-irradiation }\end{array}$} \\
\hline No HU & 0.20 & $0.12-0.27$ & 1.29 & $0.79-1.80$ & 4.30 & $2.62-5.99$ \\
\hline $\mathrm{HU}$ & 0.19 & $0.09-0.28$ & 1.23 & $1.59-1.87$ & 4.09 & $1.97-6.24$ \\
\hline
\end{tabular}

* $\mathrm{CI}=$ confidence interval.

**NM=Not meaningful; the dose response curve slope is not statistically different from zero.

The effects of SPE-like proton radiation and/or HU on the kinetics of blood cell counts determined at a dose of 2 Gy were expressed as a fraction of control for lymphocytes, granulocytes, and platelets as a function of time. The radiation dose of 2 Gy was selected because 2 Gy is the maximum deep tissue dose expected in astronauts from exposure to SPE radiation (Kim et al., 2009; Townsend, 2002). The lymphocyte counts showed a significant increase on day four after $\mathrm{HU}$ treatment alone; there were no significant changes observed at any other time points analyzed thereafter (Figure 5A, broken line). In mice exposed to radiation alone, a significant decrease of $75.0 \pm 10.7 \%$ was observed on day two postirradiation, and the lymphocyte count remained significantly lower through all time points thereafter (Figure 5A, straight line). After the 
combined treatment with irradiation and $\mathrm{HU}$, lymphocyte numbers decreased significantly on day two post-irradiation to values that were $64.0 \pm$ $8.3 \%$ lower than the sham-control value. The lymphocyte count remained significantly lower at all time points thereafter, but it was significantly higher on day 16 post-irradiation as compared to mice treated with radiation alone (Figure 5A, punctuated line).

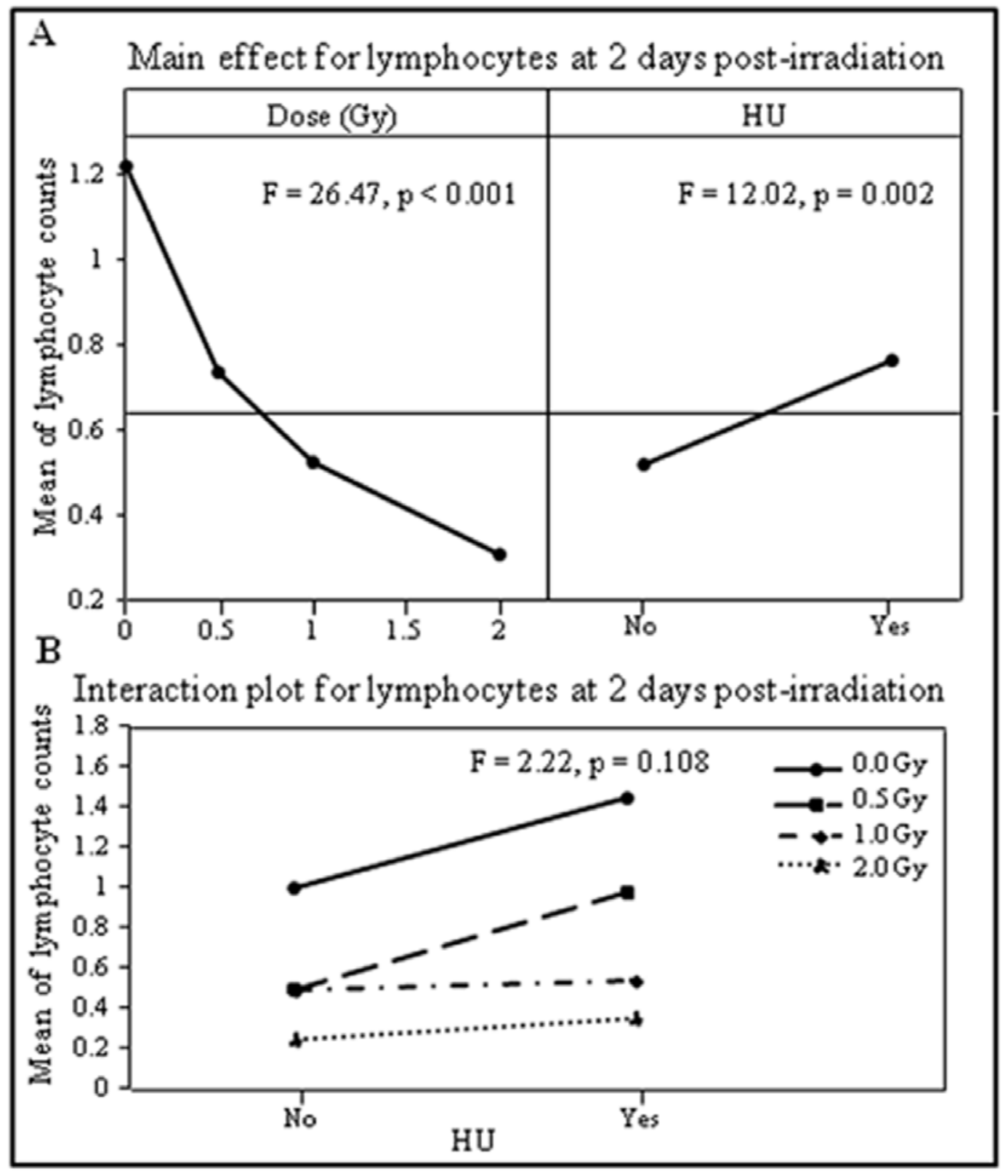

Figure 1. Mean of lymphocyte counts obtained on day two after irradiation were analyzed by DOE using a general linear model with radiation dose (Gy) and $\mathrm{HU}$ as independent variables. The results are presented in the main effects (A) and interaction (B) charts; the statistical significance of the main effects and interaction was determined by an F-test, with $p$ values shown in the respective charts. 


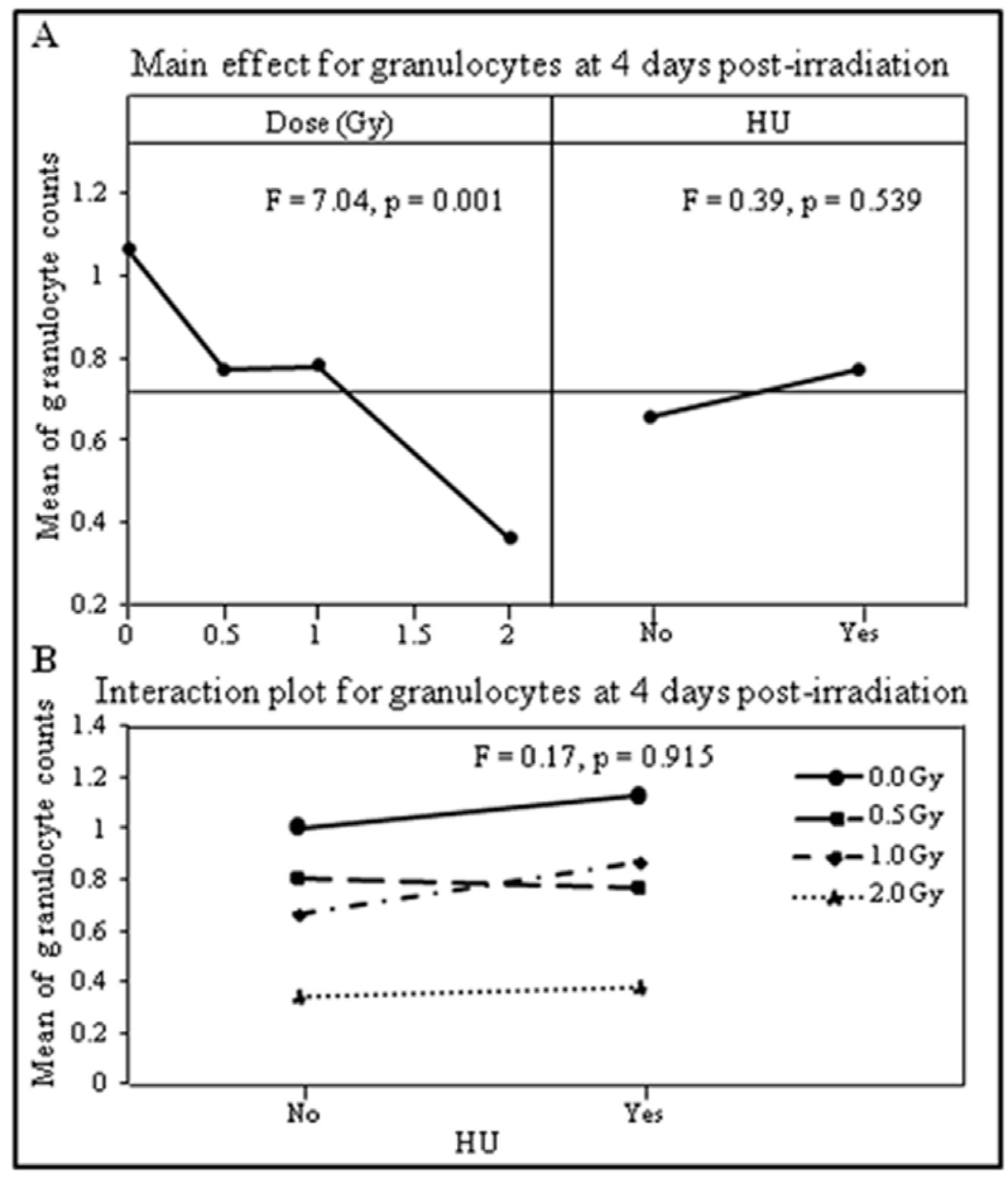

Figure 2. Mean of granulocyte counts obtained on day four after irradiation were analyzed by DOE using a general linear model with radiation dose $(\mathrm{Gy})$ and $\mathrm{HU}$ as independent variables. The results are presented in the main effects (A) and interaction (B) charts; the statistical significance of the main effects and interaction was determined by an $\mathrm{F}$-test, with $\mathrm{p}$ values shown in the respective charts.

The granulocytes exhibited no significant changes in mice treated with $\mathrm{HU}$ alone at any of the time points analyzed (Figure 5B, broken line). In mice exposed to radiation alone, the granulocyte numbers were significantly decreased on day two and day four post-irradiation by up to $66.0 \pm 14.5 \%$; they then recovered by day 10 postirradiation and then dropped significantly again by $66.7 \pm 7.2 \%$ on day 16 post-irradiation (Figure
5B, straight line). After the combined treatment, the granulocyte numbers were significantly decreased on day two and day four postirradiation; they then recovered on day 10 to numbers that were not significantly different from those of the sham-control values. On day 16 postirradiation, granulocyte counts significantly decreased again compared with the sham-controls; however, the counts were significantly higher than 
those of mice treated with radiation alone. On day four and day 16 post-irradiation, the granulocyte counts were $61.6 \pm 8.2 \%$ and $45.8 \pm 16.9$ lower than the sham-control values, respectively (Figure $5 \mathrm{~B}$, punctuated line).

No significant changes in platelet numbers were observed in animals exposed to HU at any of the time points analyzed (Figure 5C, broken line). Treatment with radiation alone caused a significant decrease in platelet number on day 10 post-irradiation, to values that were $62.0 \pm 6.6 \%$ lower than the sham-control values (Figure 5C, straight line). With the combined treatment, platelets showed a significant decrease at day 10 post-irradiation when the values were $63.5 \pm$ $11.4 \%$ less than the sham-control values (Figure $5 \mathrm{C}$, punctuated line).

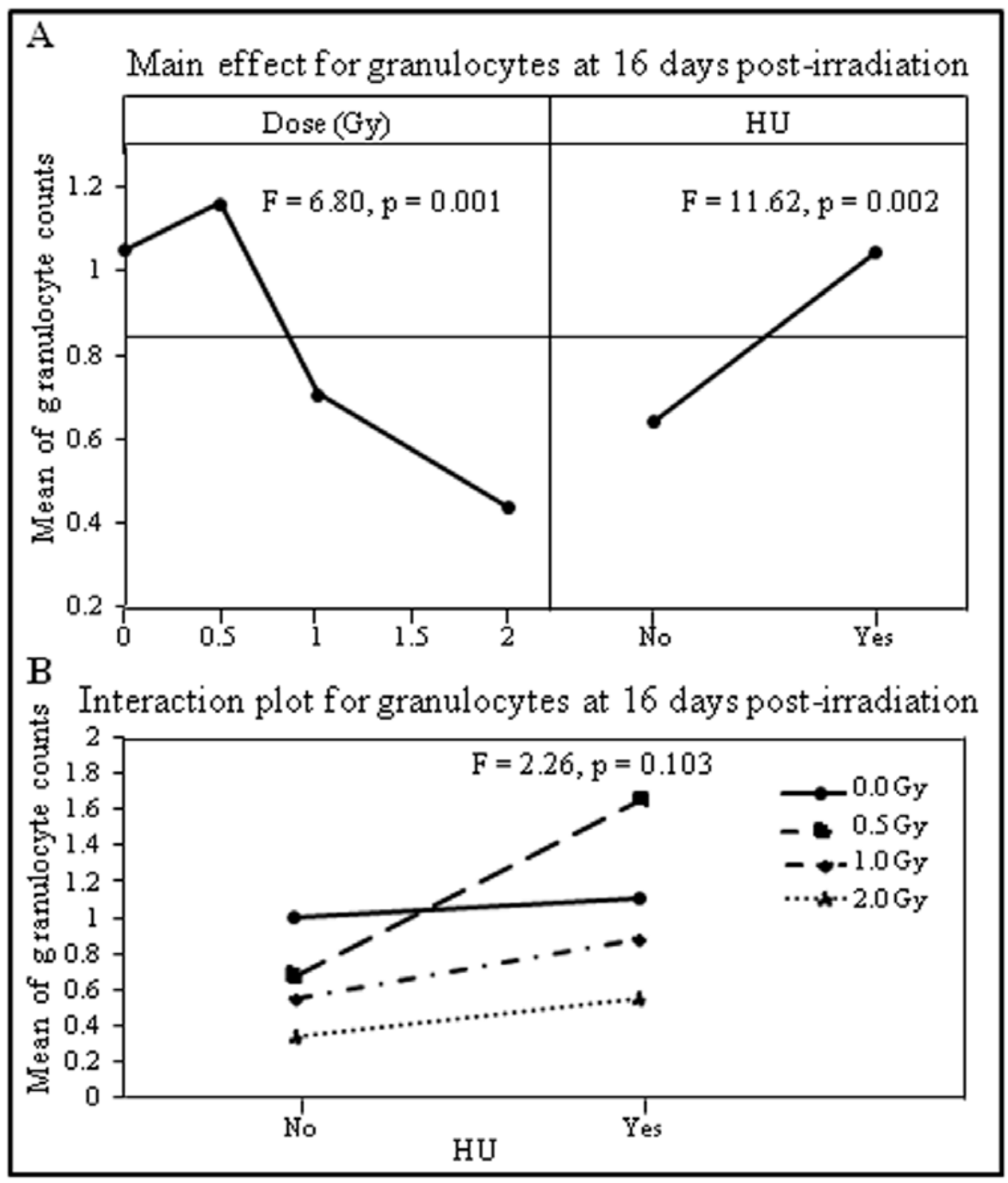

Figure 3. Mean of granulocyte counts obtained on day 16 after irradiation were analyzed by DOE using a general linear model with radiation dose (Gy) and $\mathrm{HU}$ as independent variables. The results are presented in the main effects (A) and interaction (B) charts; the statistical significance of the main effects and interaction was determined by an $F$-test, with p values shown in the respective charts. 


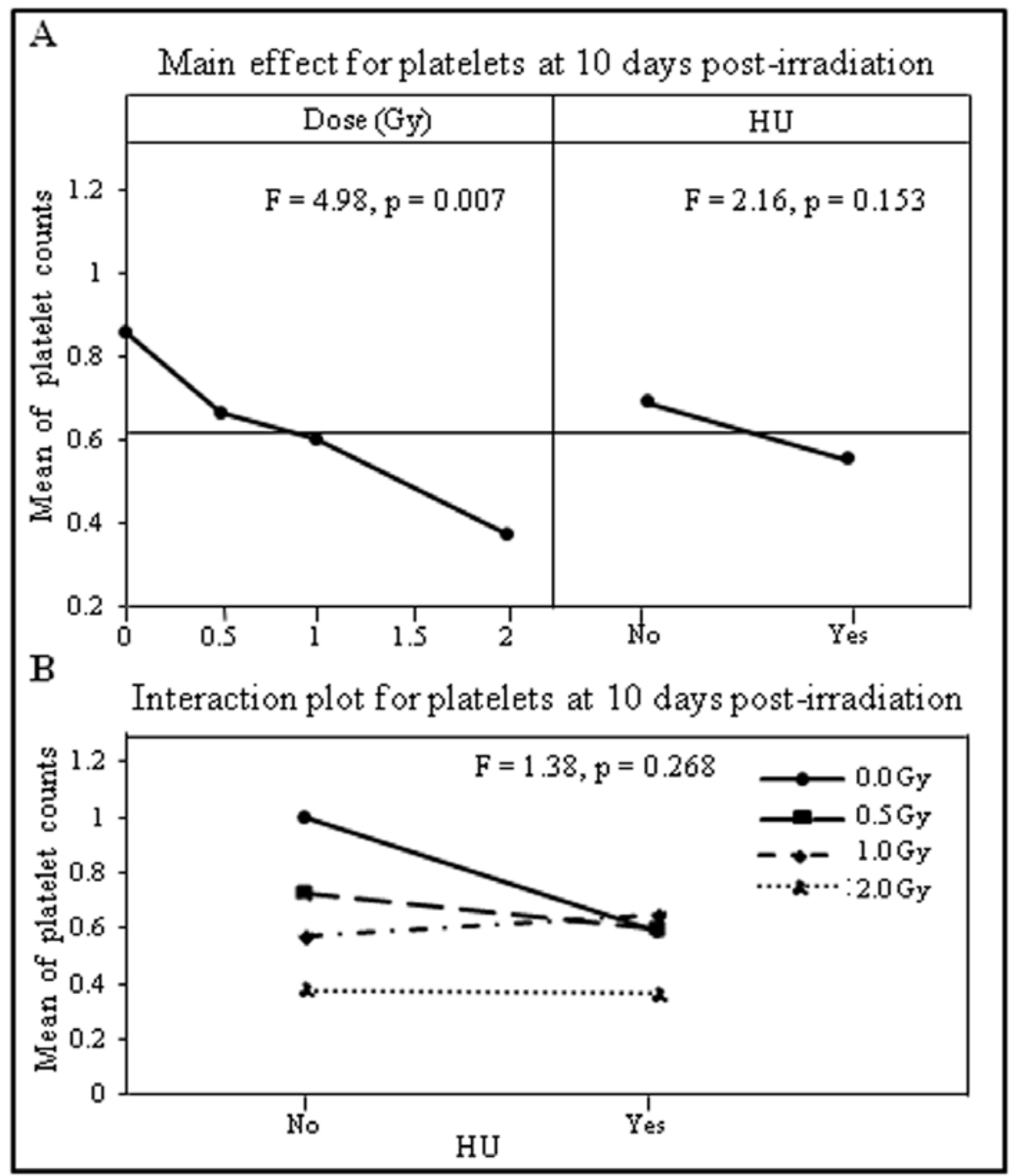

Figure 4. Mean of platelet counts obtained on day10 after irradiation were analyzed by DOE using a general linear model with radiation dose (Gy) and $\mathrm{HU}$ as independent variables. The results are presented in the main effects (A) and interaction (B) charts; the statistical significance of the main effects and interaction was determined by an $\mathrm{F}$-test, with $\mathrm{p}$ values shown in the respective charts.

\section{DISCUSSION}

One of the main stressors that astronauts encounter in space missions is microgravity. In longer and further space missions, however, they will also have a higher probability of being exposed to SPE radiation. The reported effects of reduced gravity on lymphocyte counts are inconsistent; some studies report a reduction or no change in their numbers, while others report increased numbers (Borchers et al., 2002; Allebban et al., 1994). In our studies, a significant increase in lymphocyte counts was observed in mice exposed to $\mathrm{HU}$ for four days, with no other significant changes detected at any other time point analyzed (Figure 5A, broken line).

In the case of granulocytes, most of the reports indicate increased numbers during or after spaceflight, and in studies conducted using a ground-based reduced gravity system (Borchers et al., 2002). However, there are studies that show decreased numbers of granulocytes in rats flown in space (e.g., Baqai et al., 2009; Grove et al., 1995). In our studies, a moderate increase in 




Figure 5. Effects of $\mathrm{HU}$ (broken line), proton radiation alone (straight line), and the combined treatment with radiation and $\mathrm{HU}$ (punctuated line) on mouse lymphocytes (A), granulocytes (B), and platelets (C) as a function of time. Lymphocyte, granulocyte, and platelet counts are shown as fraction of control (symbols) and standard error (error bars), and they are plotted against the time after irradiation. As indicated by the arrow, HU treatment started two days prior to irradiation. Statistical significance was indicated by $*(p<0.05)$ for a one-way ANOVA, and Tukey's test comparison of the results from each treatment group with the corresponding sham-control value, or by \# $(\mathbf{p}<0.05)$ for a $t$-test comparison of the results between the mice exposed to radiation alone and mice exposed to the combined treatment with radiation and $\mathrm{HU}$. granulocyte numbers was observed in mice exposed to HU treatment for six and 18 days; however, these changes did not reach statistical significance (Figure 5B, broken line). The effects of the combined treatment with $\mathrm{HU}$ and $2 \mathrm{~Gy}$ SPE-like proton radiation on lymphocyte, granulocyte, and platelet counts were, for the most part, similar to the effect of the treatment with 2 Gy SPE-like proton radiation alone.

We did not observe significant interaction between the radiation dose and HU treatment for effects on the lymphocyte, granulocyte, and platelet counts at the indicated nadir times, indicating that the effects of radiation and $\mathrm{HU}$ treatment are simply additive (or subtractive to be more precise) with no significant synergism. It has been reported, however, that there is a synergistic activation of the innate immune system in mice exposed to the combined treatment with 2 Gy SPE-like proton radiation and $\mathrm{HU}$, as compared with each treatment alone (Zhou et al., 2012). This apparent discrepancy can be explained by the fact that Zhou et al. measured immune system function, while in the present study the circulating number of some immune cells (lymphocytes and granulocytes) was determined. Numbers of cells are not always directly correlated with immune function, i.e., high cell numbers do not always mean increased immune function or activation.

Although the combined treatment with $\mathrm{HU}$ did not exacerbate the deleterious effects of SPElike proton radiation, the numbers of lymphocytes were still reduced by $64 \%$ or more for at least 10 days in the combined treatment group. Granulocyte numbers were reduced by $52 \%$ at the first nadir time and by $46 \%$ at the second nadir time, whereas platelet numbers were reduced by $64 \%$ at the nadir time for the combined treatment groups. The reduction in the numbers of both granulocytes and lymphocytes could compromise immune function and increase the risk of infections, while a reduction in platelet numbers might increase the risk for hemorrhagic and thrombotic diseases (Dai et al., 2009). Therefore, countermeasures may be indicated for preventing or mitigating the reduction in blood cell numbers resulting from SPE proton radiation exposure during space travel. Previously, we have demonstrated that pegfilgrastim administered subcutaneously can serve as a countermeasure for the reduction in circulating neutrophils, resulting 
from exposure of mice to 2 Gy SPE-like proton radiation (Romero-Weaver et al., 2013b). No countermeasures have been reported for the reduction in the numbers of lymphocytes and platelets after exposure to proton radiation. Thus, efforts should continue to find countermeasures for these endpoints, as well as to find agents that can be administered by an oral route, which would be easy to administer in the space environment.

\section{ACKNOWLEDGEMENTS}

This work was supported by the National Space Biomedical Research Institute (NSBRI)Center of Acute Radiation Research (CARR) grant and the NIH Radiation Biology Training Grant, 2T32CA009677. The NSBRI is funded through NASA NCC 9-58.

\section{REFERENCES}

Allebban Z, Ichiki AT, Gibson LA, Jones JB, Congdon CC, Lange RD (1994) Effects of spaceflight on the number of rat peripheral blood leukocytes and lymphocyte subsets. Journal of Leukocyte Biology 55: 209-213

Baqai FP, Gridley DS, Slater JM, Luo-Owen X, Stodieck LS, Ferguson V, Chapes SK, Pecaut MJ (2009) Effects of spaceflight on innate immune function and antioxidant gene expression. Journal of Applied Physiology 106: $1935-1942$

Borchers AT, Keen CL, Gershwin ME (2002) Microgravity and immune responsiveness: implications for space travel. Nutrition 18: 889-898

Chapes SK, Mastro AM, Sonnenfeld G, Berry WD (1993) Antiorthostatic suspension as a model for the effects of spaceflight on the immune system. Journal of Leukocyte Biology 54: $227-235$

Dai K, Wang Y, Yan R, Shi Q, Wang Z, Yuan Y, Cheng H, Li S, Fan Y, Zhuang F (2009) Effects of microgravity and hypergravity on platelet functions. Thrombosis and Haemostasis 101: 902-910

Dainiak N (2002) Hematologic consequences of exposure to ionizing radiation. Experimental Hematology 30: 513-528

Gridley DS, Rizvi A, Luo-Owen X, Makinde AY, Coutrakon GB, Koss P, Slater JM, Pecaut MJ (2008) Variable hematopoietic responses to acute photons, protons and simulated solar particle event protons. In Vivo 22: 159-169

Grove DS, Piskhak SA, Mastro AM (1995) The effect of a 10 day space flight on the function, phenotype, and adhesion molecule expression of splenocytes and lymph node lymphocytes. Experimental Cell Research 219: 102-109

Hellweg CE, Baumstark-Khan C (2007) Getting ready for the manned mission to Mars: the astronauts' risk from space radiation. Naturwissenschaften 94: 517-526

Ichiki AT, Gibson LA, Jago TL, Strickland KM, Johnson DL, Lange RD, Allebban Z (1996) Effects of spaceflight on rat peripheral blood leukocytes and bone marrow progenitor cells. Journal of Leukocyte Biology 60: 37-43

Kaur I, Simons ER, Castro VA, Ott CM, Pierson DL (2004) Changes in neutrophil functions in astronauts. Brain, Behavior, and Immunity 18: 443-450

Kaur I, Simons ER, Castro VA, Ott CM, Pierson DL (2005) Changes in monocyte functions of astronauts. Brain, Behavior, and Immunity 19: 547-554

Kim MH, Hayat MJ, Feiveson AH, Cucinotta FA (2009) Prediction of frequency and exposure level of solar particle events. Health Physics 97: 68-81

Maks CJ, Wan XS, Ware JH, Romero-Weaver AL, Sanzari JK, Wilson JM, Rightnar S, Wroe AJ, Koss P, Gridley DS, Slater JM, Kennedy AR (2011) Analysis of white blood cell counts in mice after gamma- or protonradiation exposure. Radiation Research 176: 170-176

Morey-Holton ER, Globus RK (2002) Hindlimb unloading rodent model: technical aspects. Journal of Applied Physiology 92: 1367-1377

Murdaca G, Setti M, Brenci S, Fenoglio D, Lantieri P, Indiveri F, Puppo F (2003) Modifications of immunological and neuroendocrine parameters induced by antiorthostatic bed-rest in human healthy volunteers. Minerva Medical 94: 363-378

Plett PA, Abonour R, Frankovitz SM, Orschell CM (2004) Impact of modeled microgravity on migration, differentiation, and cell cycle control of primitive human hematopoietic progenitor cells. Experimental Hematology 32: $773-781$ 
Romero-Weaver AL, Wan XS, Diffenderfer ES, Lin L, Kennedy AR (2013a) Effect of SPElike proton or photon radiation on the kinetics of mouse peripheral blood cells and radiation biological effectiveness determinations. Astrobiology 13: 570-577

Romero-Weaver AL, Wan XS, Diffenderfer ES, Lin L, Kennedy AR (2013b) Kinetics of neutrophils in mice exposed to radiation and/or granulocyte colony-stimulating factor treatment. Radiation Research 180: 177-188

Sanzari JK, Romero-Weaver AL, James G, Krigsfeld G, Liyong L, Diffenderfer ES, Kennedy AR (2013) Leukocyte activity is altered in a ground based murine model of microgravity and proton radiation exposure. PLoS One 8(8): e 71757

Sanzari JK, Wilson JM, Wagner EB, Kennedy AR (2011) The combined effects of reduced weightbearing and ionizing radiation on splenic lymphocyte population and function. International Journal of Radiation Biology 87: $1033-1038$

Townsend LW (2002) Implications of the space radiation environment for human exploration in deep space. Radiation Protection Dosimetry 115: 44-50

Wei LX, Zhou JN, Roberts AI, Shi YF (2003) Lymphocyte reduction induced by hindlimb unloading: distinct mechanisms in the spleen and thymus. Cell Research 13: 465-471

Zhou Y, Ni H, Li M, Sanzari JK, Diffenderfer ES, Lin L, Kennedy AR, Weissman D (2012) Effect of solar particle event radiation and hindlimb suspension on gastrointestinal tract bacterial translocation and immune activation. PLoS One 7: e44329 